\title{
Involvement of ERK1/2 signaling in proliferation of eight liver cell types during hepatic regeneration in rats
}

\author{
X.G. Chen ${ }^{1}$, C.S. $\mathrm{Xu}^{2,3}$ and Y.M. Liu ${ }^{1}$ \\ ${ }^{1}$ Animal Science and Technology School, \\ Henan University of Science and Technology, Luoyang, China \\ ${ }^{2}$ Key Laboratory for Cell Differentiation Regulation, Xinxiang, China \\ ${ }^{3}$ College of Life Science, Henan Normal University, Xinxiang, China \\ Corresponding author: C.S. Xu \\ E-mail: cellkeylab@126.com
}

Genet. Mol. Res. 12 (1): 665-677 (2013)

Received February 13, 2012

Accepted December 18, 2012

Published March 11, 2013

DOI http://dx.doi.org/10.4238/2013.March.11.14

\begin{abstract}
It has been well established that ERK1/2 signaling, often subdivided into nine types of pathways, can regulate the hepatocyte proliferative response during liver regeneration. However, the effect of ERK1/2 signaling on the proliferation of other hepatic cell types remains unclear. We isolated and purified 8 liver cell types at 10 time points after $2 / 3$ hepatectomy in adult rats. For each cell type, mRNA expression changes for ERK1/2 signaling-involved genes were monitored up to $168 \mathrm{~h}$, using microarrays. Real-time PCR assays were performed for array data verification. The expression levels of these genes varied considerably between different cell types. Integrating microarray results with gene synergical analysis, at the priming phase, activation of integrin/Grb2/Ras pathway in hepatocytes apparently contributed to G0/G1 transition. Two other pathways, G-protein/EPAC/Rap1 and G-protein/PKA/Rap1, were stimulated in hepatic stellate cells, while $\mathrm{RTK} / \mathrm{PKC} / \mathrm{Ras}$ and RTK/Grb2/Ras were stimulated in Kupffer cells. At the progressive phase, the ERK1/2 pathway is involved in hepatocyte replication; three pathways, namely $\mathrm{Ca}^{2+} / \mathrm{PKC} / \mathrm{Ras}, \mathrm{RTK} / \mathrm{Grb} 2 / \mathrm{Ras}$ and
\end{abstract}


G-protein/EPAC/Rap1, were found to play roles in biliary epithelial cell proliferation, while $\mathrm{RTK} / \mathrm{PKC} / \mathrm{Ras}$ and $\mathrm{RTK} / \mathrm{Grb} 2 / \mathrm{Ras}$ were involved in Kupffer cell proliferation, and G-protein/PKC/Ras in pit cell proliferation. At the terminal phase, the promotive effect of the ERK1/2 pathway on replication of hepatocytes, biliary epithelial cells, oval cells, hepatic stellate cells, Kupffer cells, and dendritic cells was considerably reduced, possibly due to their differentiation at the end of regeneration. G-protein/PKC/Ras, integrin/Grb2/Ras and G-protein/ PKA/Rap1 pathways were active in sinusoidal endothelial cells, perhaps to aid in their proliferation. We conclude that ERK1/2 has a signaling role in the regulation of proliferation of 8 cell types during liver regeneration process.

Key words: Liver restoration; Liver cell types; Gene synergy; ERK1/2 signaling pathway; Gene expression profiling

\section{INTRODUCTION}

The liver performs many crucial functions required for survival and has a relatively unique regenerative capability (Xu et al., 2010). After injury or partial hepatectomy (PH), the liver can rapidly restore itself to normal mass through hyperplasia of the remnant liver, a process called liver regeneration (LR). LR is usually segmented into a priming phase ( $0-6 \mathrm{~h}$ after $\mathrm{PH})$, a progressing phase (6-72 $\mathrm{h}$ after $\mathrm{PH})$, and a terminal phase (120-168 $\mathrm{h}$ after $\mathrm{PH}$ ) involving the activation of growth factors and cytokines, synthesis of transcription factors, reconstruction of extracellular matrix, and other processes (Ma et al., 2009). Hepatocytes (HCs) are well known to constitute the chief functional cells of the liver, responsible for delivering most of the hepatic functions important for body homeostasis, such as material storage, substance metabolism, bile secretion, oxidation protection, detoxification, biotransformation, and immune response (Xu et al., 2010). Normally, mitotic activity occurs in only $0.0012-0.01 \%$ of HCs in adult rat liver. However, under certain physiopathological stress situations such as $2 / 3$ hepatectomy or toxic injury, a large number of residual HCs can divide in response to a loss in liver mass. This process is regulated by many signaling pathways, including that of extracellular signal-related kinases 1 and 2 (ERK1/2) (Michalopoulo, 2010). For instance, Talarmin et al. (1999) have reported that partial liver resection can lead to ERK1/2 activation, which in turn enhances LR by stimulating HC proliferation. However, it is unclear which of the 9 pathways of ERK1/2 signaling (Figure 1) is involved in $\mathrm{HC}$ proliferation. Aside from $\mathrm{HCs}$, cellular components such as biliary epithelial cells (BECs), oval cells (OCs), hepatic stellate cells (HSCs), sinusoidal endothelial cells (SECs), Kupffer cells (KCs), pit cells (PCs), and dendritic cells (DCs) compose the liver. Whether ERK1/2 signaling is involved in the proliferation of these liver cells during LR and its mechanism of action if it is involved have been poorly investigated up to date.

Therefore, we used a Rat Genome 2302.0 array to detect the expression alterations of genes involved in the ERK1/2 signaling pathway in 8 liver cell types isolated from rat regenerating liver, and then analyzed the relevance of ERK1/2 signaling pathways to rat LR at the mRNA level using bioinformatics and systems biology methods, which are helpful in exploring the relevance of ERK1/2 signaling to rat LR. 


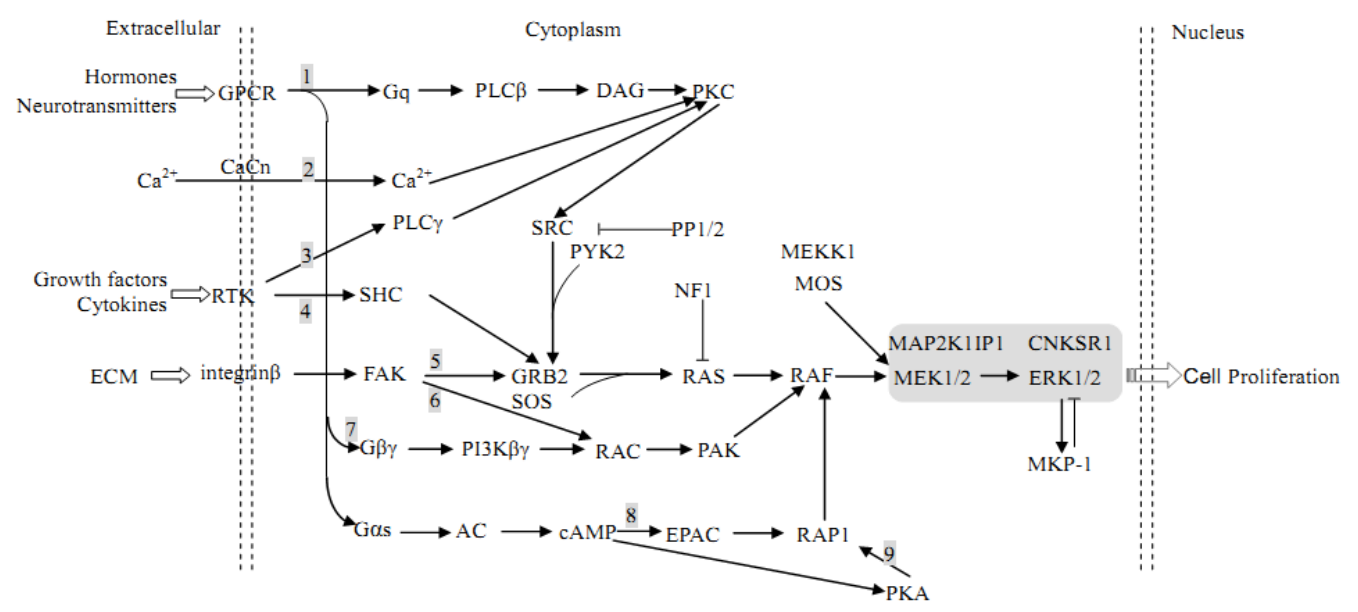

Figure 1. Diagram shows the major components of ERK1/2 signaling pathway.

\section{MATERIAL AND METHODS}

\section{Rat 2/3 hepatectomy and LR}

Sprague-Dawley rats were obtained from Henan Normal University (Animal Center of Henan Normal University, Xinxiang, Henan) in compliance with current animal protection laws in China. A total of 114 cleaning-grade Sprague-Dawley adult rats weighing $230 \pm 20 \mathrm{~g}$ were randomly divided into $9 \mathrm{PH}$ groups, 9 operation control groups, and one normal control group. Six rats were placed in each group with a ratio of males to females of 1:1. Rats in the PH groups underwent 2/3 PH surgery following a method described by Higgins and Anderson (1931). Briefly, the left and median lateral liver lobes were surgically removed, and the rats were killed $0,2,6,12,24,30,36,72,120$, and $168 \mathrm{~h}$ after PH. Rats in the operational control groups underwent the operation process, but no liver lobes were removed.

\section{Isolation and identification of liver cell types}

Rats were subjected to abdominal skin disinfection with alcohol after being anesthetized with inhaled diethyl ether. The abdominal cavity was opened to expose the liver, and the inferior vena cava was ligated below and above followed by portal vein cannulation. A conventional 2-step perfusion method was used to separate a liver cell suspension. Briefly, the liver was perfused with calcium-free perfusate preheated to $37^{\circ} \mathrm{C}$ until it turned gray. Then, 15 $\mathrm{mL} 0.05 \%$ collagenase IV solution instead of perfusate was used to perfuse the liver at a flow rate of $1 \mathrm{~mL} / \mathrm{min}$. After the liver capsule was removed, the perfused liver was cut into small pieces and digested with $0.05 \%$ collagenase IV for $15 \mathrm{~min}$ at $37^{\circ} \mathrm{C}$. After filtration through 200-well nylon netting, the liquid was centrifuged at $500 \mathrm{~g}$ for $3 \mathrm{~min}$. The pellet at the bottom was collected and washed 3 times in a $4^{\circ} \mathrm{C}$ phosphate-buffered saline (PBS) solution to adjust the cell concentration to $1 \times 10^{8}$ cells $/ \mathrm{mL}$. Six milliliters of the mixed cell suspension 
was spread onto the surface of $4 \mathrm{~mL} 60 \%$ Percoll (Pharmacia, Biotech AB, Uppsala, Sweden) in a $10-\mathrm{mL}$ tube for a single centrifugation at $200 \mathrm{~g}$ for $5 \mathrm{~min}$ at $4^{\circ} \mathrm{C}$. The centrifuged pellets and supernatant were the purified HCs and nonparenchymal cell-enriched supernatant fractions, respectively. The supernatant was mixed with an equal volume of PBS, centrifuged at $400 \mathrm{~g}$ twice for $2 \mathrm{~min}$ each time at $4^{\circ} \mathrm{C}$. The mixed nonparenchymal cell-rich pellet collected was adjusted to a concentration of $1 \times 10^{8}$ cells $/ \mathrm{mL}$ with PBS and mixed with $10 \mu \mathrm{L} / \mathrm{mL}$ rat anti-thymidylate synthase complementing protein, anti-glial fibrillary acidic protein, anticytokeratin (CK) 31, anti-cluster of differentiation (CD) 68, anti-CD161a, and anti-CD11c PE antibodies. Hepatic OCs, HSCs, SECs, KCs, PCs, and DCs were selected using a method described elsewhere (Chalmers et al., 1998). Furthermore, white intrahepatic bile duct fractions left on the nylon netting were added to the digestive solution containing $0.25 \%$ trypsin and $0.05 \%$ collagenase IV, incubated at $37^{\circ} \mathrm{C}$ for $50 \mathrm{~min}$, and filtered through 200 -well nylon netting. The filtered solution was centrifuged at $300 \mathrm{~g}$ for $5 \mathrm{~min}$. The resulting sediment was the pellet enriched with BECs. These BECs were isolated with rat anti-CK19 PE (phycoerythrin) antibody as described above. Finally, anti-albumin and glucose-6-phosphate, CK18 and gamma-glutamyltransferase 1, OC2 and OV6, CD14 and endothelin 1, lisozyme and extracellular domain 2, desmin and vimentin, CD8 and CD56, and CD86 and CD103 antibodies were used to identify HCs, BECs, hepatic OCs, HSCs, SECs, KCs, PCs, and DCs, respectively, as described elsewhere (Grisham, 1983).

\section{Microarray detection and data analysis}

Total RNA from above liver cell samples was extracted following the manual of the Trizol reagent (Invitrogen, Carlsbad, CA, USA) following manufacturer instructions and purified following the RNeasy Mini Protocol (Qiagen, Valencia, CA, USA). The quality of total RNA samples was assessed by measuring the optical density at 260/280 nm and using agarose electrophoresis $(180 \mathrm{~V}, 0.5 \mathrm{~h})$ with a $2: 1$ ratio of $28 \mathrm{~S}$ to $18 \mathrm{~S}$ ribosomal RNA intensity. As a template, $5 \mu \mathrm{g}$ total RNA was used to synthesize the first strand of cDNA using SuperScript II RT (Invitrogen) and T7-oligo dT(24) (W.M. Keck Foundation, New Haven, CT, USA) as the primer. Second-strand synthesis was performed with an Affymetrix cDNA Single-Stranded cDNA Synthesis Kit (Affymetrix Inc., Santa Clara, CA, USA). The cDNA product was purified following a cDNA purification protocol.

Twelve microliters purified cDNA served as a template for the production of a biotinlabeled cRNA transcript using the GeneChip In Vitro Transcript Labeling Kit (ENZO Biochemical, New York, NY, USA). The labeled cRNA was purified using the RNeasy Mini Kit columns (Qiagen). The concentration, purity, and quality of cDNA and cRNA were assessed as above. Fifteen microliters $1 \mu \mathrm{g} / \mu \mathrm{L} \mathrm{cRNA}$ was incubated with $6 \mu \mathrm{L} 5 \mathrm{X}$ fragmentation buffer and $9 \mu \mathrm{L}$ RNase-free water for $35 \mathrm{~min}$ at $94^{\circ} \mathrm{C}$ and digested into 35- to 200-bp cRNA fragments. The prehybridized Rat Genome 2302.0 microarray was put into a hybridization buffer prepared following the Affymetrix protocol and hybridized in a rotating chamber $(60 \mathrm{rpm}$, $16 \mathrm{~h}, 45^{\circ} \mathrm{C}$ ). Arrays were washed to remove the superfluous hybridization buffer, stained in a GeneChip Fluidics Station 450 (Affymetrix Inc.), and scanned with a GeneChip Scanner 3000 (Affymetrix Inc.) to obtain images. The images were converted to signal values using the Affymetrix GCOS 1.4 software (Affymetrix Inc.).

The probe signal values were scaled to evaluate gene expression $(\mathrm{P}<0.05)$, marginal 
expression $(\mathrm{P}<0.065)$, and no expression $(\mathrm{P}>0.065)$. Then, the signal value of each chip was normalized, and the relative values of genes were assessed with ratios comparing the normalized $\mathrm{P}$ value in the $\mathrm{PH}$ groups to that in controls. For example, gene expression with a relative value of $\geq 3$ was considered to be upregulated, gene expression with a relative value of $\leq 0.33$ was considered to be downregulated, and genes with a relative expression value of 0.33-2.99 were considered to be meaningless genes (Vardhanabhuti et al., 2006). To minimize technical errors from microarray analysis, we analyzed each sample at least 3 times with the Rat Genome 2302.0 microarray. Average values were calculated as a corrective value. Finally, these values were analyzed using GeneMath, GeneSpring (Silicon Genetics, San Carlos, CA, USA) and other programs (Mulrane et al., 2008).

\section{Real-time polymerase chain reaction (RT-PCR)}

The RNA samples used for RT-PCR were prepared from 8 kinds of liver cells isolated at 10 recovery time points. cDNA synthesis was performed according to the manufacturer protocol. Primer and probe sequences were designed using Primer Express 2.0 according to mRNA sequences of HC marker glucose-6-phosphatase- $\alpha$, BEC marker gamma-glutamyltransferase 1, OC marker $O C 2$, HSC marker glial fibrillary acidic protein, SEC marker CD14, $\mathrm{KC}$ marker lisozyme, PC marker CD56, and DC marker CD86 and synthesized by Shanghai GeneCore BioTechnologies Co., Ltd. (Shanghai). Target genes were subjected to amplification and PCR testing on a PRISM 7900 Sequence Detector (ABI Company, USA) according to the operational guideline manual with a QuantiTect SYBR Green RT-PCR Kit (Qiagen). The copies of target genes in the sample per milliliter were calculated according to a standard curve, and the relative expression content was computed according to $\beta$-actin copy number (Wang and $\mathrm{Xu}, 2010$ ).

\section{RESULTS}

\section{Identification of ERK1/2 signaling-involved genes}

According to receptor types of ERK1/2, such as G-protein, calcium ion channel, receptor tyrosine kinase (RTK) and integrin, ERK1/2 signaling was roughly divided into 4 pathways: G-protein-mediated ERK1/2, $\mathrm{Ca}^{2+}$-mediated ERK1/2, RTK-mediated ERK1/2, and integrin-mediated ERK1/2. Despite the variations in their specific extracellular signals, these pathways had some common components, including a set of adaptors (Shc, GRB2, Crk, etc.) linking the receptor to a guanine nucleotide exchange factor (SOS, C3G, etc.) for transducing the signals to small guanosine triphospate binding proteins (Ras, Ras-proximate-1), which in turn stimulated mitogen-activated protein kinase (MAPK) kinase kinase (Raf), MAPK kinase (MEK1/2) and MAPK (RK1/2). Genes involved in these pathways were obtained by checking available signaling pathways in databases including the Rat Genome Database, Gene MicroArray Pathway Profiler, Kyoto Encyclopedia of Genes and Genomes, BIOCARTA, and Biocompare. These genes were then reconfirmed through retrieval of pertinent articles. We found that the number of genes involved in these 4 signaling pathways were 79, 50, 44, and 30 , respectively (Table 1). 


\section{Table 1. Overview of ERK1/2 signaling pathway-involved genes.}

\begin{tabular}{|c|c|c|c|c|c|c|c|c|c|c|}
\hline \multirow[t]{2}{*}{ Pathways } & \multirow[t]{2}{*}{ Involved genes } & \multirow[t]{2}{*}{ Genes on arrays } & \multicolumn{8}{|c|}{ Liver regeneration-related genes } \\
\hline & & & $\mathrm{HC}$ & $\mathrm{BEC}$ & $\mathrm{OC}$ & HSC & SEC & $\mathrm{KC}$ & $\mathrm{PC}$ & DC \\
\hline G-protein-mediated ERK1/2 pathway & 79 & 77 & 24 & 27 & 12 & 34 & 21 & 17 & 20 & 30 \\
\hline $\mathrm{Ca}^{2+}$-mediated ERK1/2 pathway & 50 & 48 & 16 & 16 & 10 & 19 & 13 & 14 & 13 & 20 \\
\hline RTK-mediated ERK1/2 pathway & 44 & 41 & 13 & 11 & 6 & 19 & 12 & 12 & 7 & 15 \\
\hline Integrin-mediated ERK1/2 pathway & 30 & 29 & 6 & 6 & 2 & 6 & 9 & 5 & 4 & 6 \\
\hline Total* & 165 & 161 & 59 & 55 & 32 & 64 & 41 & 39 & 44 & 69 \\
\hline
\end{tabular}

$\mathrm{HC}=$ hepatocytes BEC $=$ biliary epithelial cells; $\mathrm{OC}=$ oval cells; $\mathrm{HSC}=$ hepatic stellate cells; $\mathrm{SEC}=$ sinusoidal endothelial cells; $\mathrm{KC}=$ Kupffer cells; $\mathrm{PC}=$ pit cells; $\mathrm{DC}=$ dendritic cells. ${ }^{*}$ Two or more signal pathways shared one gene.

\section{Reliability of chip results by quantitative RT-PCR}

To test the reliability of the chip data, we measured the expression levels of 8 marker genes corresponding to the 8 selected liver cell types using RT-PCR. The comparison analysis of the RT-PCR results and the microarray results showed no significant difference in abundances or trends of gene expression obtained from the 2 methods, demonstrating the reliability of the chip results (Figure 2).
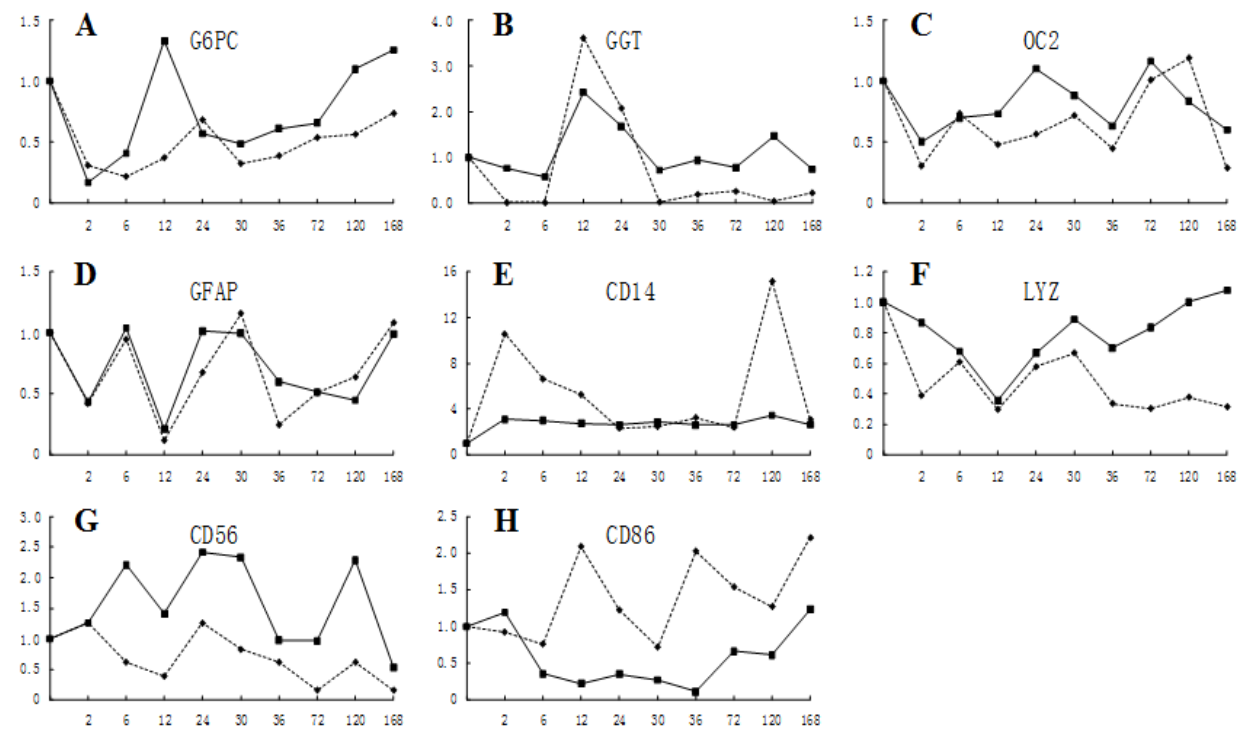

Figure 2. Comparison of mRNA levels of cell marker genes measured by microarray (solid lines) and RT-PCR (broken lines) in 8 liver cell types including hepatocytes (A), biliary epithelial cells (B), oval cells (C), hepatic stellate cells (D), sinusoidal endothelial cells $(\mathbf{E})$, Kupffer cells $(\mathbf{F})$, pit cells $(\mathbf{G})$, and dendritic cells $(\mathbf{H})$. Vertical axis indicates the relative mRNA level; horizontal axis indicates the recovery time points after $2 / 3$ hepatectomy. 


\section{Global characteristics of transcriptional profiles of ERK1/2 signaling pathway genes in 8 cell types during rat LR}

The Rat Genome 2302.0 array used in this study encompassed the entire rat genome. Array hybridizations were performed in triplicate for each sample. The relevant data retrieved from signaling pathway databases and scientific publications were compared with chip results, showing that $77,48,41$, and 29 genes were on chip that were separately involved in G-protein-, $\mathrm{Ca}^{2+}$-, RTK-, and integrin-mediated ERK1/2 pathways, respectively (see Table 1). Among these genes, those showing 1) the same expression patterns at the same time point in 3 independent analyses, 2) at least a 3-fold change in expression level compared to that in the control for at least one time point, and 3$)$ significant $(\mathrm{P} \leq 0.05)$ or extremely significant $(\mathrm{P} \leq$ 0.01 ) differences between the $\mathrm{PH}$ and sham-operated groups, were identified based on stringent standards as LR-related genes. By interrogating the microarray results of 8 rat liver cell types, we found that many genes did not conform to the criteria for defining LR-related genes and that a large difference in expression changes in these genes occurred from one cell type to another. The 4 pathway-involved genes conforming to the above standard in HCs, BECs, OCs, HSCs, SECs, KCs, PCs, and DCs are shown in Table 1.

To visualize the temporal expression patterns of genes across the 8 rat liver cell types after $\mathrm{PH}$, we performed $\mathrm{H}$-clustering analysis. The results suggested that ERK1/2 signalinginvolved genes were clustered in the confined regions in the heat map (Figure 3). For example, a majority of genes involved in the G-protein-mediated ERK1/2 pathway were upregulated during LR in HCs, SECs, and PCs (Figure 3A); $\mathrm{Ca}^{2+}$-mediated ERK1/2 pathway-involved genes were largely upregulated in HCs, BECs, PCs, and DCs but downregulated in HSCs (Figure 3B). RTK-mediated ERK1/2 pathway-involved genes showed markedly reduced expression in HSCs (Figure 3C). Integrin-mediated ERK1/2 pathway-involved genes showed significantly increased expression in HCs and BECs (Figure 3D).

\section{Differential expressions of gene involved in 4 ERK1/2 signaling pathways in 8 rat liver cell types}

An overall analysis of various online databases showed that the above 4 ERK1/2 pathways acted through 9 different specific pathways. As shown in Figure 3, the G-proteinmediated ERK1/2 pathway can be subdivided into the G-protein/protein kinase C (PKC)/ Ras (pathway 1), G-protein/phosphoinositide-3-kinase (PI3K)/Rac (pathway 7), G-protein/EPAC (exchange protein directly activated by cyclic AMP)/Ras-proximate-1 (Rap1) (pathway 8), and G-protein/PKA (protein kinase A)/Rap1 (pathway 9) pathways. The $\mathrm{Ca}^{2+}-$ mediated ERK1/2 pathway comprises only $\mathrm{Ca}^{2+} / \mathrm{PKC} / \mathrm{Ras}$ (pathway 2); the RTK-mediated ERK1/2 pathway contains RTK/PKC/Ras (pathway 3) and RTK/growth factor receptorbound protein 2 (Grb2)/Ras (pathway 4), and the integrin-mediated ERK1/2 pathway is classified into integrin/Grb2/Ras (pathway 5) and integrin/focal adhesion kinase/Rac (pathway 6). The number of genes (which were presented in array) involved in the nine signaling pathways were $47,48,38,30,24,21,31,20,25,2,19,1,42$, and 1, respectively (Figure 4). However, different cell types varied in expression changes and the number of genes involved in the above signal pathways. 


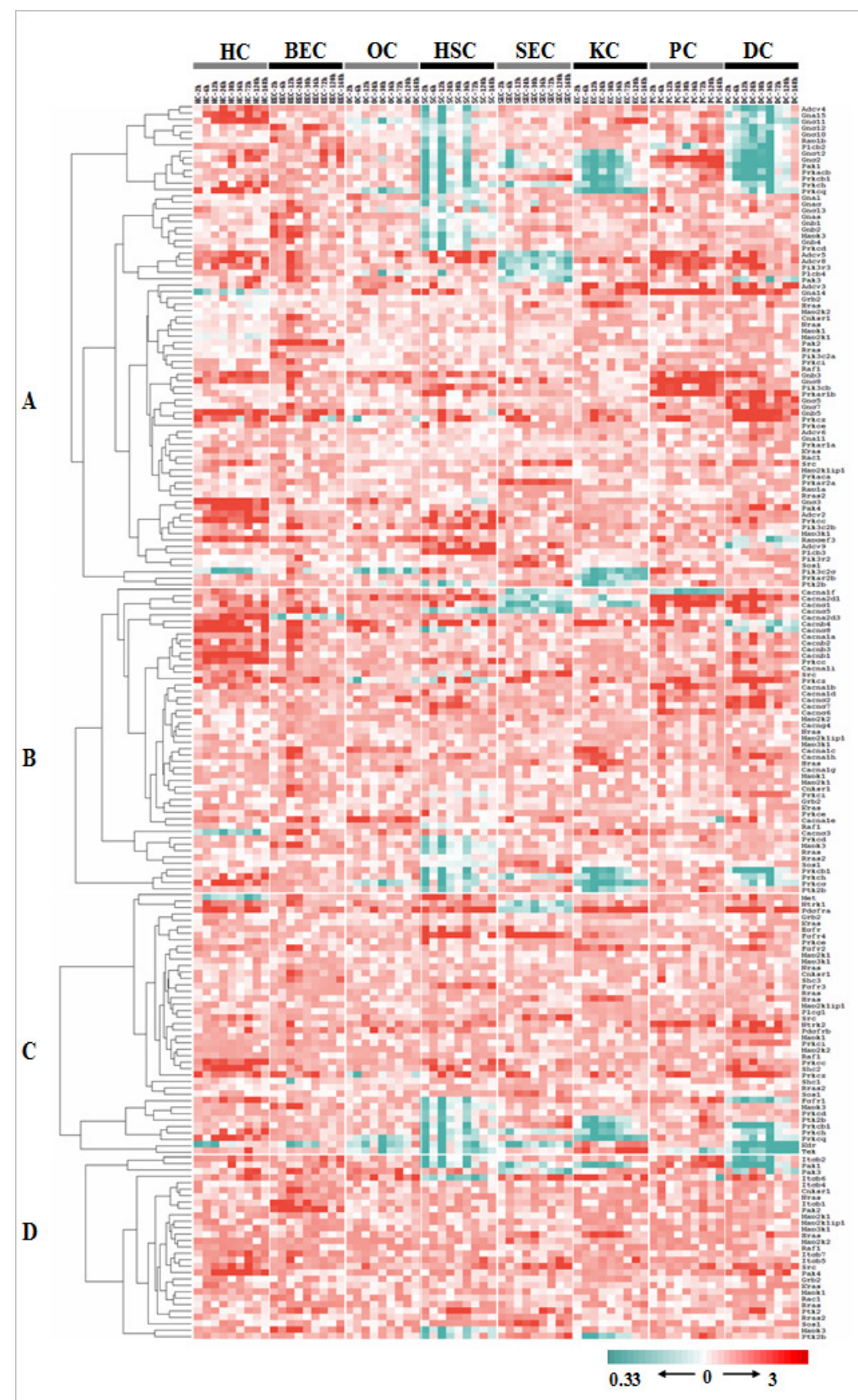

Figure 3. Hierarchical clustering of the genes of ERK1/2 signaling pathways in 8 rat liver cell types after partial hepatectomy. Two heat maps A, B, C, D, and E represented the gene clusters related to G-protein mediated, $\mathrm{Ca}^{2+}$-mediated, RTK-mediated, intergrin-mediated ERK1/2 cascade, and regulatory pathway. Numbers oriented horizontally at the top indicate the recovery time points post-surgery. The increase, decrease and meaningless changes in expression level were colored in red, blue and white, respectively. For abbreviations, see legend to Table 1. 


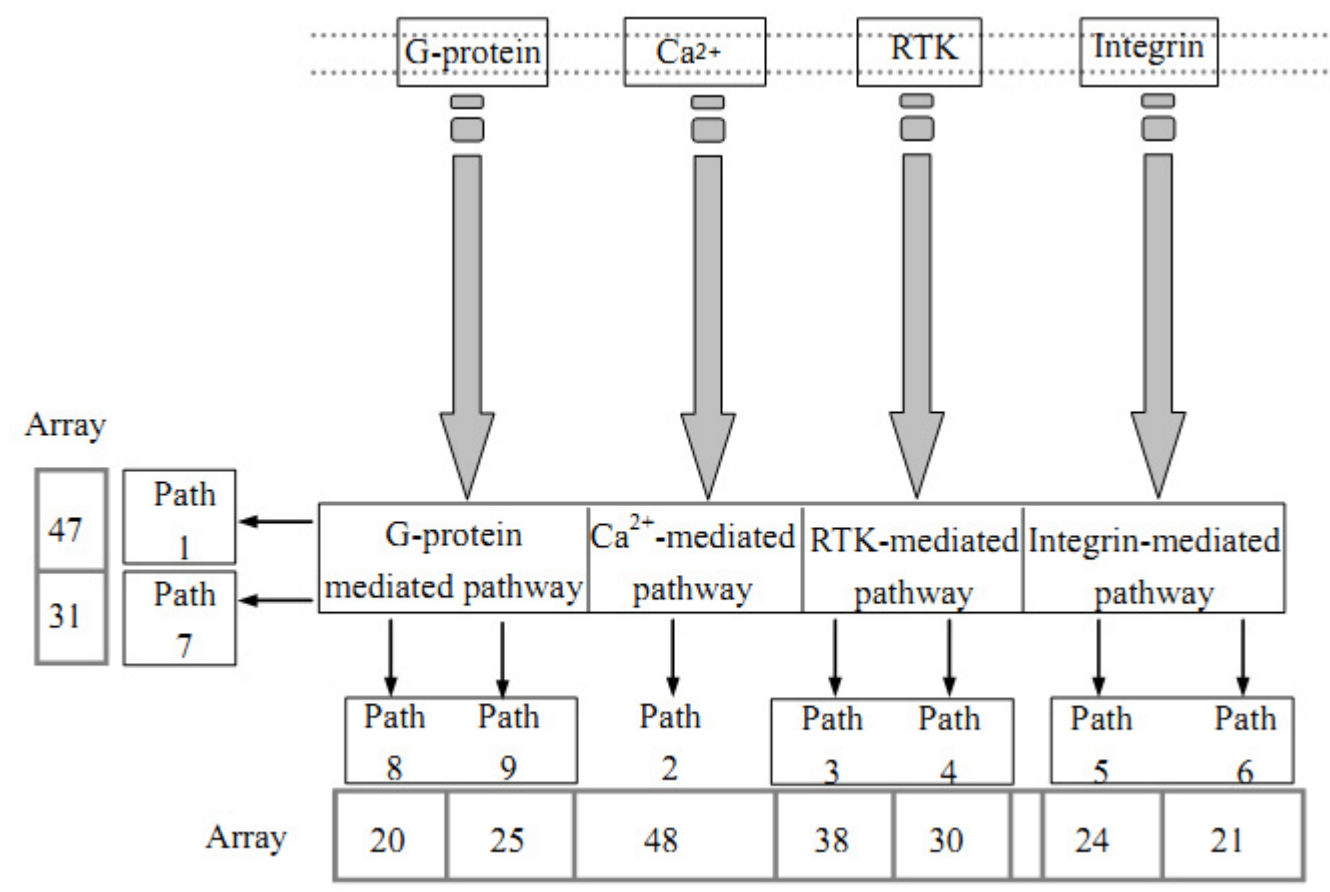

Figure 4. Schematic representations of 9 ERK1/2 signaling pathways and number of the related genes detected by microarray. Path $1=$ G-protein $\rightarrow \mathrm{PKC} \rightarrow$ Ras; Path $2=\mathrm{Ca}^{2+} \rightarrow \mathrm{PKC} \rightarrow \mathrm{Ras}$; Path $3=\mathrm{RTK} \rightarrow \mathrm{PKC} \rightarrow \mathrm{Ras}$; Path $4=$ RTK $\rightarrow$ Grb2 $\rightarrow$ Ras; Path $5=$ integrins $\rightarrow$ Grb2 $\rightarrow$ Ras; Path $6=$ integrins $\rightarrow$ FAK $\rightarrow$ Rac; Path $7=$ G-protein $\rightarrow$ PI3K $\rightarrow$ Rac; Path $8=$ G-protein $\rightarrow$ EPAC $\rightarrow$ Rap1; Path $9=$ G-protein $\rightarrow$ PKA $\rightarrow$ Rap 1.

\section{Physiological activities governed by ERK1/2 signaling in 8 liver cell types from hepatectomized rats}

Based on gene ontology categories, transcript abundance, expression dynamics, and the correlation (Nikitin et al., 2003) among genes in certain specific biological process during LR, a mathematical model based on multivariate statistical analyses (Wang and Xu, 2010) was established for depicting the timing, occurrence, and intensity of biological activities in LR using time sequence analysis theory and systematic biology methodologies (Wolkenhauer, 2001). Depending on this model, the synergy among the components involved in ERK1/2 signaling in each cell type was calculated. The results showed that, as a whole, ERK1/2 signaling was only slightly affected at the priming phase (2-6 h after $\mathrm{PH})$, whereas the G-protein-mediated, RTK-mediated, and integrin-mediated pathways were strikingly enhanced in $\mathrm{HCs}$, and the G-protein-, $\mathrm{Ca}^{2+}$, and RTK-mediated pathways were dominated by activation in BECs, SECs, KCs, and PCs at the progressing phase (6-72 h after PH). Most of the ERK1/2 signaling pathway activations gradually decreased and returned to normal or became even weaker than those in the control - e.g., G-protein-, RTK-, and integrin-mediated ERK1/2 pathways in DCs (Table 2). 


\begin{tabular}{|c|c|c|c|c|c|c|c|}
\hline \multirow[t]{2}{*}{ Liver cell types } & \multirow[t]{2}{*}{ ERK $1 / 2$ signaling pathways } & \multicolumn{2}{|c|}{ Initial phase } & \multicolumn{2}{|c|}{ Proliferative phase } & \multicolumn{2}{|c|}{ Terminal phase } \\
\hline & & $E_{t}$ & $\overline{\text { Effects }}$ & $E_{t}$ & $\overline{\text { Effects }}$ & $E_{t}$ & Effects \\
\hline \multirow[t]{7}{*}{$\mathrm{HC}$} & 1 & 52.5 & - & 66.0 & $\uparrow *$ & 72.8 & - \\
\hline & 3 & 50.2 & - & 67.8 & $\uparrow * *$ & 76.8 & - \\
\hline & 5 & 25.7 & - & 34.1 & $\uparrow * *$ & 36.0 & - \\
\hline & 6 & 16.3 & - & 22.6 & $\uparrow * *$ & 25.3 & - \\
\hline & 7 & 28.3 & - & 37.0 & $\uparrow * *$ & 40.4 & - \\
\hline & 8 & 24.3 & - & 34.8 & $\uparrow * *$ & 37.2 & - \\
\hline & 9 & 24.3 & - & 32.0 & $\uparrow * *$ & 33.0 & - \\
\hline \multirow[t]{4}{*}{$\mathrm{BEC}$} & 2 & 26.9 & - & 34.5 & $\uparrow * *$ & 25.6 & - \\
\hline & 4 & 58.4 & - & 67.8 & $\uparrow *$ & 57.1 & - \\
\hline & 8 & 21.6 & _- & 25.6 & $\uparrow *$ & 19.0 & _- \\
\hline & 9 & 26.4 & - & 29.5 & - & 23.5 & $\downarrow^{*}$ \\
\hline $\mathrm{OC}$ & 1 & 70.5 & $\downarrow^{*}$ & 74.7 & - & 77.6 & - \\
\hline \multirow[t]{3}{*}{$\mathrm{HSC}$} & 7 & 23.6 & - & 25.6 & $\downarrow^{*}$ & 26.8 & - \\
\hline & 8 & 17.7 & $\uparrow *$ & 17.5 & - & 18.3 & - \\
\hline & 9 & 17.0 & $\uparrow *$ & 17.1 & - & 18.0 & - \\
\hline \multirow[t]{4}{*}{ SEC } & 1 & 47.4 & $\downarrow *$ & 52.6 & - & 54.3 & $\uparrow *$ \\
\hline & 5 & 29.6 & - & 35.8 & - & 38.2 & $\uparrow *$ \\
\hline & 7 & 27.6 & - & 30.3 & $\downarrow^{*}$ & 31.7 & - \\
\hline & 9 & 20.3 & - & 22.9 & $\uparrow^{*}$ & 24.4 & $\uparrow^{*}$ \\
\hline \multirow{2}{*}{ KC } & 3 & 53.5 & $\uparrow *$ & 54.6 & $\uparrow *$ & 56.9 & - \\
\hline & 4 & 44.5 & $\uparrow *$ & 45.0 & $\uparrow *$ & 44.9 & - \\
\hline \multirow[t]{2}{*}{ PC } & 1 & 49.4 & - & 55.6 & $\uparrow *$ & 56.6 & $\uparrow *$ \\
\hline & 7 & 33.4 & - & 32.7 & - & 32.9 & $\uparrow *$ \\
\hline \multirow[t]{7}{*}{$\mathrm{DC}$} & 1 & 58.1 & - & 57.6 & - & 42.7 & $\downarrow *$ \\
\hline & 4 & 47.1 & - & 44.8 & - & 33.2 & $\downarrow *$ \\
\hline & 5 & 19.2 & - & 20.4 & - & 19.7 & $\downarrow *$ \\
\hline & 6 & 17.6 & - & 18.7 & - & 19.0 & $\downarrow *$ \\
\hline & 7 & 29.2 & - & 28.8 & - & 25.1 & $\downarrow *$ \\
\hline & 8 & 18.2 & - & 16.9 & - & 16.0 & * \\
\hline & 9 & 23.0 & - & 21.5 & - & 19.9 & * \\
\hline
\end{tabular}

*Significant difference; **extremely significant difference; $(\uparrow)=$ activity is stronger than control; $(\downarrow)=$ activity is weaker than control. 1. G-protein $\rightarrow \mathrm{PKC} \rightarrow \mathrm{Ras} ; 2$. $\mathrm{Ca}^{2+} \rightarrow \mathrm{PKC} \rightarrow \mathrm{Ras} ; 3$. RTK $\rightarrow \mathrm{PKC} \rightarrow \mathrm{Ras} ; 4$. RTK $\rightarrow \mathrm{Grb} 2 \rightarrow \mathrm{Ras}$; 5. integrins $\rightarrow$ Grb2 $\rightarrow$ Ras; 6. integrins $\rightarrow$ FAK $\rightarrow$ Rac; 7. G-protein $\rightarrow$ PI3K $\rightarrow$ Rac; 8. G-protein $\rightarrow$ EPAC $\rightarrow$ Rap 1; 9. G-protein $\rightarrow$ PKA $\rightarrow$ Rap 1 . For liver cell type abbreviations, see legend to Table 1.

\section{DISCUSSION}

This study detected the expression profiles of genes involved in ERK1/2 signaling in 8 liver cell types after rat $\mathrm{PH}$ and then comparatively analyzed the proliferation activities predicted by expression changes using bioinformatics and systems biology methods. We found that a total of 9 ERK1/2 signaling pathways have roles in the regulation of cell proliferation.

The early period of 2-6 $\mathrm{h}$ after $\mathrm{PH}$ is often called the priming phase, and the main events occurring in this stage are the activation of $\mathrm{HCs}$, which enter G1 from G0 during which the active nuclear factor kappa B signaling pathway plays a major role (Liu and Qian, 2006). Whether other signal pathways such as ERK1/2 contribute to cell activation remains poorly studied. Talarmin et al. (1999) have reported that ERK1/2 activation at 0.5-4 h after PH is responsible for the priming of quiescent HCs, with transition from G0 to G1. In the present study, gene synergy analysis showed that the proliferation-promoting integrin/Grb2/Ras pathway in HCs was more active than that in controls, and at the same time, Src in this pathway showed increased expression with a peak 4.7-fold higher than that in the control $6 \mathrm{~h}$ after PH, implying that Src may play a key role in this event. In addition, the proliferation-promoting G- 
protein/EPAC/Rap1 and G-protein/PKA/Rap1 pathways in HSCs were significantly enhanced in our study. Balabaud et al. (2004) have suggested that HSCs are activated at the early phase, and activation lasts until at least $24 \mathrm{~h}$ post-PH. However, these authors do not point to the specific signaling pathway responsible for HSC stimulation. In the context of our results, we speculate that ERK1/2 may participate in HSC activation via the above-mentioned G-protein/ EPAC/Rap1 pathway and G-protein/PKA/Rap1 pathway. Also, gene synergy analysis showed that the proliferation-promoting RTK/PKC/Ras and RTK/Grb2/Ras pathways in KCs were activated, and theoretically, the stimulation of the 2 pathways was implicated in KC activation. Despite a lack of data addressing the timing of KC activation, Desbois-Mouthon et al. (2006) reported that KCs activated after hepatectomy can secrete and release a bulk of cytokines and growth factors (including tumor necrosis factor $\alpha$, hepatocyte growth factor, epidermal growth factor) promoting $\mathrm{G} 0 / \mathrm{G} 1$ transition of $\mathrm{HCs}$ suggests that the timing of $\mathrm{KC}$ activation is close to that of HC. Based on our analysis, the enhancement of RTK/PKC/Ras and RTK/Grb2/Ras pathway activities was helpful for $\mathrm{KC}$ activation after $\mathrm{PH}$.

The period of 6-72 $\mathrm{h}$ after $\mathrm{PH}$ is defined as the progressing phase of LR, during which HCs enter the G1 phase of the cell cycle in response to growth factors and progress. DesboisMouthon et al. (2006) have reported that ERK1/2 signaling is associated with the induction of cyclins A, D1, E, and B1 between 10 and $11 \mathrm{~h}$ in the G1/S transition phase. In this study, almost all of the proliferation-promoting REK1/2 pathways showed increased HC activities during the progressing phase, inferring the promotion of the cell cycle of HCs through this pathway, which is consistent with the results of Desbois-Mouthon et al. (2006).

Notably, various liver cell populations displayed great differences in peak DNA replication, which occurred $36 \mathrm{~h}$ after PH in BECs but $24 \mathrm{~h}$ after PH in HCs (Magami et al., 2002). Our study showed that the proliferation-promoting $\mathrm{Ca}^{2+} / \mathrm{PKC} / \mathrm{Ras}, \mathrm{RTK} / \mathrm{Grb} 2 / \mathrm{Ras}$, and G-protein/EPAC/Rap1 pathways in BECs augmented activation - all 3 pathways may be involved in BEC replication. As mentioned above, the activities of the G-protein/EPAC/Rap1 and G-protein/PKA/Rap1 pathways in HSCs during the priming phase showed insignificant difference compared to that of the control during the progressing phase, whereas the proliferation-promoting G-protein/PI3K/Rac pathway was enhanced at this time. Michalopoulos (2010) have found that DNA replication of HSCs after PH peaks $44-48 \mathrm{~h}$ after surgery. Obviously, our result was inconsistent with theirs, which infers that ERK1/2 signaling may not be involved in HSC proliferation. SECs, one type of nonparenchymal cells, displayed delayed replication. DNA synthesis in SECs is completed within $96 \mathrm{~h}$ after PH (Clavien et al., 2007), which is beyond the usual definition of the progressing phase (6-72 $\mathrm{h}$ after $\mathrm{PH})$. Therefore, we propose that ERK1/2 signaling has a very small role in SEC proliferation. According to our data, the proliferation-promoting G-protein/PI3K/Rac pathway in SECs was decreased, whereas the G-protein/PKA/Rap1 pathway increased, and the regulatory effects of the 2 pathways on SEC proliferation are opposed, consistent with a minor role for ERK1/2. Regarding the proliferation dynamics of 3 additional liver immune cells (KCs, PCs, and DCs), as far as we know, DNA replication reaches a peak $48 \mathrm{~h}$ post-PH in KCs; for PCs, the peak occurs 24-72 h after PH (Xu et al., 2011), and the timing for DCs is unclear. In this study, the RTK/ $\mathrm{PKC} / \mathrm{Ras}$ and RTK/Grb2/Ras pathways in KCs were activated during the progressing phase, and these 2 pathways likely contribute to $\mathrm{KC}$ repopulation at this time. Gene synergy of the G-protein/PKC/Ras pathway in PCs was significantly higher than that of the control, and the consistency between the activation time of this pathway and the timing of PC proliferation clearly showed that these pathways are involved in PC proliferation. 
The 120- to 168 -h period after $\mathrm{PH}$ is considered the terminal phase, during which HCs cease growth and start differentiation for the rebuilding of tissue structure (Liu and Qian, 2006). BECs, HSCs, and KCs also begin to differentiate at this stage, implying that the ERK $1 / 2$ signal pathway no longer has a positive effect on the proliferation of these cells. Our analysis showed that the synergistic effect of ERK1/2 signaling-involved genes in these liver cell types was approximately equal to or even lower than that in the control - a result concordant with the hypothesis of decreased ERK1/2 effect. As for stem cells in the liver, or OCs, the activity of ERK $1 / 2$ signaling had no noticeable effect in comparison with controls at the terminal phase. Previously, we observed the impairment of OC proliferation $72 \mathrm{~h}$ after surgery. The consistency between that result and those of the present study reinforces the conclusion that the growth and proliferation of OCs are blocked at the end of LR. As described above, SEC proliferation peaks $96 \mathrm{~h}$ after $\mathrm{PH}$ (Clavien et al., 2007), which occurs within the terminal phase and logically assumes the involvement of ERK1/2 signaling in SEC replication. Unanimously, proliferation-promoting pathways including G-protein/PKC/Ras, integrins/Grb2/Ras, and Gprotein/PKA/Rap1 were enhanced at this phase, inferring the positive regulation of SEC proliferation by ERK1/2 signaling via the 3 pathways. Of note, only the adenylate cyclase type 3 gene in pathway 9 displayed a markedly increased expression with a peak 3 -fold higher than that in controls $6 \mathrm{~h}$ after $\mathrm{PH}$, perhaps implying the importance of the adenylate cyclase type 3 in the activity of the G-protein/PKA/Rap1 pathway. Based on the observation that PC proliferation occurs between 24 and $74 \mathrm{~h}$ after hepatectomy, we propose that the major events occurring in PCs is differentiation, during which the effect of ERK1/2 on PC proliferation would be obviously weakened. However, our results showed a striking increase in synergistic effects of the proliferation-promoting G-protein/PKC/Ras and G-protein/PI3K/Rac pathways during the terminal phase compared to that in the control, which does not support our hypothesis. According to above analysis, these 2 signaling pathway likely have no promotive effect on cell proliferation but rather influence other biological effects. Finally, the synergistic effect of Erk1/2 signaling in DCs was much lower than that in the control, which was helpful to DC differentiation at the end of LR.

In conclusion, this study measured the transcriptional profiles of ERK1/2 signalinginvolved genes using a Rat Genome 2302.0 array and obtained many detailed but accurate data concerning ERK1/2 signaling in 8 liver cell types. These data suggest the involvement of 9 pathways of ERK1/2 signaling in the proliferation of these cell types after $\mathrm{PH}$ in rats. However, a Rat Genome 2302.0 array can measure only gene transcription profiles and cannot reflect protein synthesis. Therefore, we would use methods such as gene addition, RNA interference, and protein interaction in future studies that test the above results.

\section{ACKNOWLEDGMENTS}

Research supported by the National Basic Research 973 Pre-Research Program of China (\#2010CB534905).

\section{REFERENCES}

Balabaud C, Bioulac-Sage P and Desmoulière A (2004). The role of hepatic stellate cells in liver regeneration. J. Hepatol. 40: $1023-1026$.

Chalmers JJ, Zborowski M, Sun L and Moore L (1998). Flow through, immunomagnetic cell separation. Biotechnol. Prog. 14: 141-148. 
Clavien PA, Petrowsky H, DeOliveira ML and Graf R (2007). Strategies for safer liver surgery and partial liver transplantation. N. Engl. J. Med. 356: 1545-1559.

Desbois-Mouthon C, Wendum D, Cadoret A, Rey C, et al. (2006). Hepatocyte proliferation during liver regeneration is impaired in mice with liver-specific IGF-1R knockout. FASEB J. 20: 773-775.

Grisham JW (1983). Cell types in rat liver cultures: their identification and isolation. Mol. Cell Biochem. 53-54: 23-33.

Higgins GM and Anderson RM (1931). Experimental pathology of the liver: restoration of the liver of the white rat following partial surgical removal. Arch. Pathol. 12: 186-202.

Liu ZG and Qian YB (2006). Regulation of liver regeneration. Med. Recapitulate 12: 332-335.

Ma XM, Dong XS and Zhao HL (2009). Research progress of liver regeneration-related molecules. Int. J. Surg. 36: $760-763$

Magami Y, Azuma T, Inokuchi H, Kokuno S, et al. (2002). Cell proliferation and renewal of normal hepatocytes and bile duct cells in adult mouse liver. Liver 22: 419-425.

Michalopoulos GK (2010). Liver regeneration after partial hepatectomy: critical analysis of mechanistic dilemmas. Am. J. Pathol. 176: 2-13.

Mulrane L, Rexhepaj E, Smart V, Callanan JJ, et al. (2008). Creation of a digital slide and tissue microarray resource from a multi-institutional predictive toxicology study in the rat: an initial report from the PredTox group. Exp. Toxicol. Pathol. 60: 235-245.

Nikitin A, Egorov S, Daraselia N and Mazo I (2003). Pathway studio - the analysis and navigation of molecular networks. Bioinformatics 19: 2155-2157.

Talarmin H, Rescan C, Cariou S, Glaise D, et al. (1999). The mitogen-activated protein kinase kinase/extracellular signalregulated kinase cascade activation is a key signalling pathway involved in the regulation of $\mathrm{G}(1)$ phase progression in proliferating hepatocytes. Mol. Cell Biol. 19: 6003-6011.

Vardhanabhuti S, Blakemore SJ, Clark SM, Ghosh S, et al. (2006). A comparison of statistical tests for detecting differential expression using Affymetrix oligonucleotide microarrays. OMICS 10: 555-566.

Wang GP and Xu CS (2010). Reference gene selection for real-time RT-PCR in eight kinds of rat regenerating hepatic cells. Mol. Biotechnol. 46: 49-57.

Wolkenhauer O (2002). Systems biology: the reincarnation of systems theory applied in biology. Brief Funct. Genomic Proteomic 2: 258-270.

Xu C, Chen X, Chang C, Wang G, et al. (2010). Transcriptome analysis of hepatocytes after partial hepatectomy in rats. Dev. Genes Evol. 220: 263-274.

Xu CS, Chen XG, Chang CF, Wang GP, et al. (2011). Differential gene expression and functional analysis of pit cells from regenerating rat liver. Genet. Mol. Res. 10: 678-692. 